\title{
Test Method of Laser Detection Sensitivity Based on Every Pulse Measurement and Rearrangement
}

\author{
Hao Guo, Na Ma, Hongpeng Zhao, Mei Yao \\ Luoyang Electronic Equipment Test Center of China, Luoyang, China \\ Email: whatbeam@163.com
}

How to cite this paper: Guo, H., Ma, N., Zhao, H.P. and Yao, M. (2017) Test Method of Laser Detection Sensitivity Based on Every Pulse Measurement and Rearrangement. Optics and Photonics Journal, 7, 33-39.

https://doi.org/10.4236/opj.2017.78B006

Received: April 28, 2017

Accepted: August 7, 2017

Published: August 10, 2017

\begin{abstract}
It is very important to accurately measure the detection sensitivity of laser receiving equipment. Based on the traditional test method of detection probability curve, a new test method is proposed which works through measuring laser pulse one by one. Accurate measurement systems were constructed to improve the accuracy of laser energy measurement and energy regulation. A new data processing method of detection probability curve is put forward, which based on subsection statistics. The new data processing method in effect reduces the light source instability from $6.57 \%$ to $0.67 \%$. These works improve the test accuracy of laser receiving detection sensitivity. It is a great support for the accurate evaluation of key technical indices of laser receiving equipment, which subsequently are done by models and simulation.
\end{abstract}

\section{Keywords}

Laser Detection, Sensitivity, Instability, Pulse Rearrangement

\section{Introduction}

Some key technical indices of laser receiving device such as scatter intercept radius and working distance, is closely related to detection sensitivity. When testing and appraising the performance of laser receiving device, it is firstly recommended to test detection sensitivity, then to simulate and evaluate the key technical indices such as scatter intercept radius and working distance, using mathematic model. This method combining test with model has many prominent merits, such as being independent of outside conditions, saving time and money, etc. It is also able to evaluate the use effect in different situation and varying weather. These varying test and evaluation are conducive to a comprehensive 
and accurate assessment of the performance of laser receiving equipment. Above of all, it's extremely important to test the detection sensitivity accurately.

\section{Present Situation and Improvement of Sensitivity Test}

Traditional test method of detection sensitivity is a direct test method [1] [2] [3], you can adjust the laser power density received by laser receiving device through changing attenuation, until the detection probability is equal to specified value. Now the current laser power density is detection sensitivity. But traditional test method have two disadvantages: the first is finite adjusting range and precision of laser power density, which caused by attenuation devices, the second is the difficulty to look after both test precision and speed.

Ma et al. put forward a test method of detection sensibility based on detection probability curve. By changing the attenuation in ascending order, you can make detection probability to decrease gradually from $100 \%$ to $0 \%$. Meanwhile, it's needed to measure the laser power density received by laser receiving device. It's recommended to use beam splitter when without high sensitivity power meter. As a result, you can get a curve. The horizontal axis is incident laser power density, the ordinate is detection probability. Then you can get the detection sensitivity from the curve through the specified detection probability.

Ma's method [4] is superior to the traditional test method, but it need a high stability laser at least. Usually the output pulse energy/power instability standard deviation of solid laser fluctuates between $5 \%$ and $10 \%$. It may not be able to largely avoid the error of detection probability caused by laser instability.

To further improve the accuracy of the laser receiving detection probability curve test, we can start from two aspects: construction of accurate measurement devices and improvement of measuring method. The main technical measures includes four aspects:

1) Construction of high stability laser. The output energy instability of 1.06 $\mu \mathrm{m}$ pulse laser is usually 5\% - 10\% (RMS), few can reach 3\% - 5\% (RMS), but it is difficult to further increase the laser stability in short term.

2) Possessing of high sensitive measurement capability. The two is a high sensitive measurement of weak laser. Due to the high sensitivity of the laser receiving equipment, it generally requires the laser power measuring device with high sensitivity in test. There are few commercial laser probe available today, so it requires specialized fabrication.

3) Possessing of precise continuous fast attenuation capability.

Besides the high stability laser source, precise continuous fast attenuation capability is also needed to fully meet the rapid and accurate detection probability curve measurement requirements. The traditional absorption attenuation slice combination mode has low adjusting speed and finite step. It needs new method to realize precise continuous fast attenuation, for example, polarization attenuation.

4) Innovation of measuring method and data processing method. In order to 
accurately measure the laser receiving detection probability curve, it needs not only accurate measurement instruments, but also innovative design on method. It's necessary to innovate measuring method and data processing method.

Therefore, in order to further improve measurement accuracy, this paper presents a new method of measuring pulse on detection probability curve one by one.

\section{Test Method}

The new test method of laser detection probability curve mainly includes test system construction, test layout, test process and so on.

\subsection{Test System Construction}

The measuring equipments needed in new test method include $1.06 \mu \mathrm{m}$ pulse laser, precise continuous attenuator, high sensitivity laser energy meter, precise timing measuring equipment, laser diffuse reflection plate, fast photodetector, oscilloscope and so on.

$1.06 \mu \mathrm{m}$ pulse laser provides light source to test. Precise continuous attenuator adjusts the laser energy illuminating laser receiving deivce. High sensitivity laser energy meter is used to measure the laser power density at the laser receiving device. Accurate timing measurement device can real-time measure relative time information of laser pulse and laser waring device outputs. Laser diffuse reflection board can reflect laser beam, forming a wide coverage on receiving window. Fast photodetector and oscilloscope is used to measure laser pulse width.

Traditional method uses beam splitting and calibrating to finish test. New test method can finish test detectly with precise equipment mentioned above.

\subsection{Test Layout}

The test layout of new test method was shown in Figure 1.

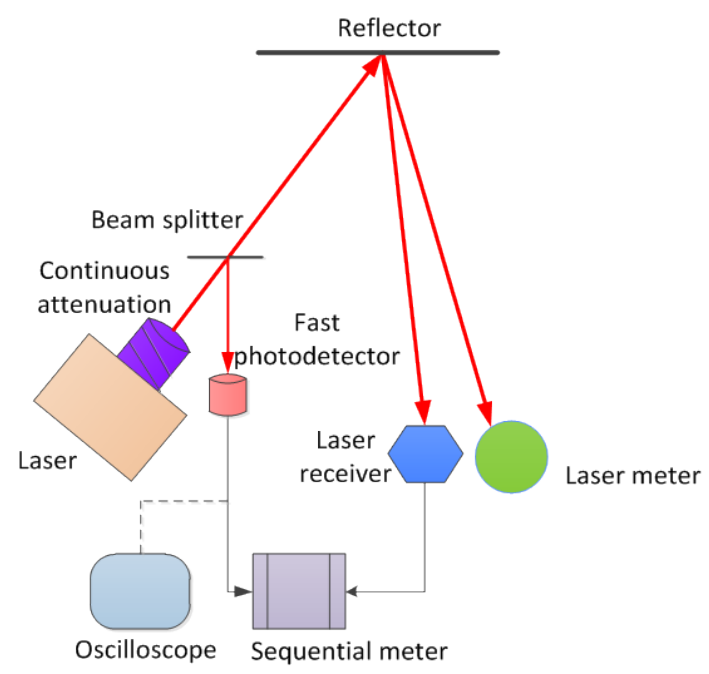

Figure 1. Schematic diagram of test. 
Laser receiving device was leveled and placed on the normal of reflection board when testing. High sensitivity laser energy meter had the same position with laser waring device, aiming at the center of reflection board. $1.06 \mu \mathrm{m}$ pulse laser aimed at the center of reflection board too. Precise continuous attenuator was lay in front of pulse laser.

One input channel of accurate timing measurement device was connected with fast photodetector to measure every transmitted pulse's arriving time. Another input channel of accurate timing measurement device was connected with the output of laser receiving device to measure warning pulse's arrival time.

\subsection{Equations}

1) All test devices were powered and booted. Each device was loaded suitable working parameters. Laser transmitted laser pulse, and other equipments received corresponding signals. Laser receiving equipment received laser pulse and sent out warning rusult of every pulse. High sensitivity laser energy meter measured every pulse's energy density. Precise timing measurement equipment measured the numbers and timing of transmitted pulse and warning pulse. Meanwhile, detection probability was counted.

2) Changing the attenuation with the appropriate step and repeating test, we can make detection probability to decrease gradually from $100 \%$ to $0 \%$, during which the ratio named $c$ between the variation named $d p$ and the variation named $\mathrm{dA}$ remains unchanged. Here $\mathrm{dp}$ is the variation of detection probability of the adjacent test points, $\mathrm{dA}$ is the variation of attenuation. The relation among them can be shown as Formula (1).

$$
\frac{d p}{d A}=c
$$

3) Laser pulse width $\tau$ was measured by fast photodetector and oscilloscope.

\subsection{Data Processing Method}

1) Laser energy density can be converted to power density by Formula (2).

$$
P=\frac{E}{\tau}
$$

2) Relationship is established between the measured power density of each transmitted laser pulse and the corresponding alarm result (whether alarm).

3) All the pulse power density are arranged in ascending order, formating a longer data space. With the appropriate principle, he long data space is divided into a plurality of adjacent sub space. The detection probability is counted in each sub space, and the power density of each subspace is averaged.

4) The statistics data is processed by nonlinear regression analysis with error function. The regression parameters are calculated. Regression equation, confidence and regression effect are counted and evaluated. Detection probability curve is drawn. The regression equation is shown as Formula (3). 


$$
P_{d}=0.5+0.5 \operatorname{erf}(a P-b)
$$

5) The laser power density at the specified detection probability can be predicted by the regression equation, so the detection sensitivity is obtained.

\section{Comparison of Test Results}

Compared with the traditional test method, the main innovation of this method is to reduce the error greatly caused by the output jitter of the light source.

According to the standard rules [5], pulse laser output energy instability can be calculated as this: laser pulse energy Qi is measured $n(n \geq 10)$ times in same interval. Average $\mathrm{Q}$ is calculated. We can find out the maximum value and minimum value of $\mathrm{Qmax}, \mathrm{Qmin}$. Energy variation $\Delta \mathrm{Q}$ and energy standard deviation $Q \sigma$ can be obtained according to the Formula (4) and Formula (5).

$$
\begin{aligned}
& \Delta Q=Q_{\text {max }}-Q_{\min } \\
& Q_{\sigma}=\sqrt{\frac{\sum_{i=1}^{n}\left(Q_{i}-Q\right)^{2}}{n-1}}
\end{aligned}
$$

Laser output energy instability SQ and output energy instability standard deviation $\delta \mathrm{Q} \sigma$ can be counted according to the Formula (6) and Formula (7).

$$
\begin{gathered}
S_{Q}= \pm \frac{\Delta Q}{2 Q} \times 100 \% \\
S_{Q \sigma}=\frac{Q_{\sigma}}{Q} \times 100 \%
\end{gathered}
$$

Onetest results are disposed respectively by traditional method and this method. The disposed results are shown in Table 1 .

The following Figure 2 is an instability comparison of 800 laser pulses energy density disposed by traditional method and this method.

From above analysis, we can find out that laser output energy instability is reduced from $6.57 \%$ to $0.67 \%$ by this method. The final result is equivalent to using a very stable laser source, the influence of laserinhibitory is restrained effectively.

Table 1. Comparison of the results disposed by traditional method and this method.

\begin{tabular}{ccc}
\hline \multirow{2}{*}{ Item Name } & \multicolumn{2}{c}{ Method } \\
\cline { 2 - 3 } & Traditional Method & This Method \\
\hline $\mathrm{Q}_{\max }$ & 3.4170 & 3.2900 \\
$\mathrm{Q}_{\min }$ & 2.9880 & 3.2460 \\
$\Delta \mathrm{Q}$ & 0.4290 & 0.0440 \\
$\mathrm{Q}$ & 3.2647 & 3.2662 \\
$\mathrm{Q}_{\sigma}$ & 0.0639 & 0.0131 \\
$\mathrm{~S}_{\mathrm{Q}}$ & $\pm 6.57 \%$ & $\pm 0.67 \%$ \\
$\mathrm{~S}_{\mathrm{Q} \sigma}$ & $1.96 \%$ & $0.40 \%$ \\
\hline
\end{tabular}




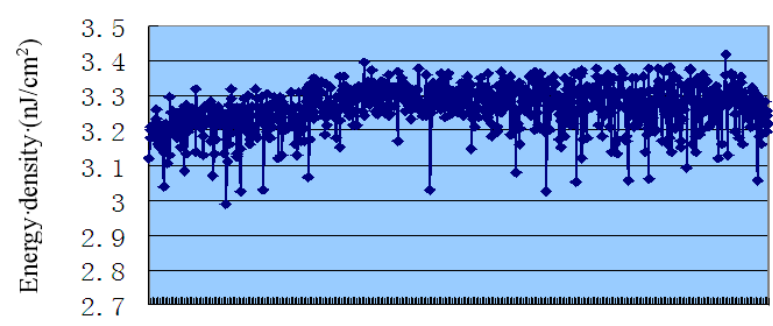

$1 \quad 69137205273341409477545613681749$

Pulse numbers

(a)

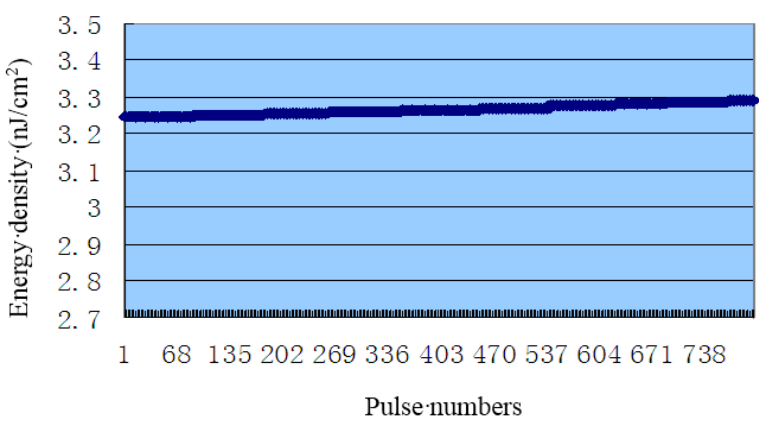

(b)

Figure 2. Instability comparison of traditional method and this method. (a) Original instability: $6.57 \%$; (b) Instability disposed by this method: $0.67 \%$.

\section{Conclusions}

Compared with the traditional test method, the innovation of this paper is mainly embodied in the following two points:

1) This method uses precise timing measurement equipment to measure pulse emission time and alarm information simultaneously, the corresponding relationship is established between each transmitted pulse and alarm result.

2) Using the method of subsection statistics, all test data are rearranged and segmented, the unfavorable influence of laser output energy source instability is effectively restrained.

These methods above can effectively improve the test accuracy of the laser detection probability curve, and provide a basis for the subsequent use of mathematical simulation methods to accurately evaluate the key technical indicators, such as the radius of interception and the working distance.

\section{References}

[1] Su, Y.G., Zhong, J., Tian, X.M., Sun, Y. and Zhu, M. (1999) General Specification for Laser Reconnaissance and Warning Device. $C N G J B, 3684-3699$.

[2] Wang, X. and Zhang, Y.K. (2013) Accurate Measurement Technology of Laser Detection Sensitivity. Electro-Optic Technology Application, 28, 78-80.

[3] Yang, X.J., Zhou, B., Ying, J.J. and Zhou, Z.L. (2010) Test Method of Detection Sensitivity of Starring Laser Detecting Systems. Laser Technology, 34, 132-134.

[4] Ma, N., Ding, Z.D. and Lei, P. (2009) New Test Mode of Detection Sensitivity Based 
on Probability Curve. Laser \& Infrared, 39, 1142-1145.

[5] Zhang, P., Qiu, Y., Guo, Z. and Zhao, X.C. (2005) Measuring Methods for SolidState Lasers. $C N G J B, 5441-2005$.

\section{Scientific Research Publishing}

Submit or recommend next manuscript to SCIRP and we will provide best service for you:

Accepting pre-submission inquiries through Email, Facebook, LinkedIn, Twitter, etc. A wide selection of journals (inclusive of 9 subjects, more than 200 journals)

Providing 24-hour high-quality service

User-friendly online submission system

Fair and swift peer-review system

Efficient typesetting and proofreading procedure

Display of the result of downloads and visits, as well as the number of cited articles

Maximum dissemination of your research work

Submit your manuscript at: http://papersubmission.scirp.org/

Or contact opj@scirp.org 